\title{
Prescrito Versus Infundido em Pacientes Críticos com Alimentação Enteral Exclusiva
}

\section{Prescribed Versus Infused in Critical Patients with Exclusive Enteral Feeding}

\author{
Mirela Ribeiro Santos Silva ${ }^{1}$ \\ Adriana Gomes Cézar Carvalho² \\ Luciana Maria Martinez $\mathrm{Vaz}^{3}$
}

\section{RESUMO}

Objetivos: A presente pesquisa teve como objetivo avaliar o volume prescrito versus o volume infundido em pacientes críticos com alimentação enteral exclusiva e identificar as devidas interrupções da infusão da dieta, como os gastos gerados devido às essas interrupções. Metodologia: Trata-se de um estudo observacional, prospectivo, realizado com pacientes internados na Unidade de Terapia Intensiva de Hospital Universitário, no período de julho a setembro de 2019, na qual inclui pacientes adultos e idosos. A amostra foi composta por 27 pacientes, internados na UTI geral, de ambos os sexos, tendo sido utilizado um questionário que abordou informações pessoais, triagem nutricional, acompanhamento dietético, intercorrências, motivos de pausa e custo da administração da dieta, coletados por meio do prontuário. Os dados foram avaliados quanto à normalidade por meio do teste de normalidade de Shapiro-Wilk, considerando um nível de significância estatística de 0,05 $(p<0,05)$. Resultados: Constatou-se que os valores encontrados do volume prescrito versus o volume infundido não foram estatisticamente significativos. A maioria dos pacientes apresentou ao menos uma intercorrência, sendo a diarreia, os exames e os procedimentos médicos ou de enfermagem as que apresentaram uma maior frequência. $O$ gasto médio desses pacientes no período de internação foi de R\$ 3.221,62. Conclusão: Esse estudo contribui para mostrar a importância do trabalho integrado da equipe multidisciplinar com o paciente crítico, visando melhorar o resultado do tratamento, minimizando as complicações, assim como o tempo de permanência na UTI, na qual influenciará em menores custos para o hospital.

\section{DESCRITORES}

Unidades de Terapia Intensiva. Terapia Nutricional. Nutrição Enteral.

\begin{abstract}
Objectives: The present study aimed to assess the prescribed volume versus the infused volume in critically ill patients with exclusive enteral feeding, and to identify the appropriate interruptions in the infusion diet, such as the expenses generated due to these interruptions. Methodology: This was an observational, prospective study with patients admitted to the Intensive Care Unit of the University Hospital, from July to September 2019 , in which includes adults and elderly patients. The sample consisted of 27 patients, admitted to the general ICU and of both sexes, with a questionnaire that addressed personal information, nutritional screening, diet monitoring, complications, reasons for pausing and the cost of administering the diet, collected through the medical records. Data were assessed for normality using the Shapiro-Wilk's normality test, considering a level of statistical significance of $0.05(p<0.05)$. Results: It was found that the values for the prescribed volume versus the infused volume were not statistically significant. Most patients had at least one complication, such as diarrhea, medical or nursing procedures, which have the highest frequency. The average expense of these patients during the hospital stay was $R \$ 3,221.62$. Conclusion: This study contributes to show the importance of the integrated work of the multidisciplinary team with the critical patient, aiming to improve the result of treatment, minimizing complications, as well as the length of stay in the ICU, which will influence lower costs for the hospital.
\end{abstract}

\section{DESCRIPTORS}

Intensive Care Units. Nutritional Therapy. Enteral Nutrition.

\footnotetext{
${ }^{1}$ Nutricionista, Residente do Programa de Pós-Graduação modalidade Residência Multiprofissional em Saúde Hospitalar com ênfase em Paciente Crítico do Hospital Universitário Lauro Wanderley - Universidade Federal da Paraíba/UFPB. Paraíba, Brasil.

${ }^{2}$ Nutricionista, Mestra, Universidade Federal da Paraíba/UFPB. Paraíba, Brasil.

${ }^{3}$ Nutricionista, Doutoranda, Universidade Federal da Paraíba/UFPB. Paraíba, Brasil
} 
A desnutrição é definida como o estado resultante da deficiência de nutrientes que podem causar alterações na composição corporal, funcionalidade e estado mental, com prejuízo no desfecho clínico. Pode ser causada por fatores de privação alimentar, doenças, idade avançada, de forma isolada ou combinada ${ }^{1}$.

No ambiente hospitalar a desnutrição é um fato frequente e muitas vezes negligenciado. Tem como principais complicações: aumento no tempo de internação, piora no sistema imunológico, demora no processo de cicatrização, aumento do risco de complicações cirúrgicas, de infecções, de lesão por pressão, de mortalidade e, portanto, dos custos hospitalares ${ }^{2}$.

Os pacientes em Unidade de Terapia Intensiva (UTI) possuem resposta inflamatória aguda que envolve o hipermetabolismo. As reservas de carboidratos são insuficientes para suprir a elevada demanda energética, forçando assim, o organismo a obter energia por meio da degradação das moléculas de proteínas, para utilização na neoglicogenese, gerando um balanço nitrogenado negativo e, consequentemente, a perda da massa muscular ${ }^{3}$.

As principais diretrizes recomendam que a terapia nutricional para pacientes críticos deve ser iniciada precocemente e de forma adequada e, com isso, diminuindo o déficit energético e, consequentemente, atenuando as alterações metabólicas presentes, bem como o catabolismo. É favorável utilizar, sempre que possível, o trato digestório. Sendo assim, a nutrição enteral precoce é indicada como estratégia nutricional para esses paciente ${ }^{2}$.
Segundo as diretrizes da American Society for Parenteral and Enteral Nutrition $(\mathrm{ASPEN})^{4}$, a terapia nutricional precoce tem o seu início nas primeiras 24 a 48 horas, após a admissão do paciente, seja ela hospitalar, na UTI ou de alguma abordagem cirúrgica. Esse início pode variar devido o estado geral do mesmo e pela presença de estabilidade hemodinâmica ${ }^{4}$.

A terapia nutricional enteral (TNE) tem alguns benefícios como: manutenção do estado nutricional, que consequentemente reduz o tempo de hospitalização e a diminuição da morbidade e mortalidade ${ }^{5}$. A escolha do acesso enteral vai depender do tempo de duração previsto para a terapia, do grau de risco de deslocamento da sonda ou risco de aspiração do paciente, das condições do trato gastrintestinal e de alterações anatômicas ${ }^{6}$.

A adequada nutrição do paciente é uma estratégia terapêutica proativa. É possível identificar problemas nutricionais, minimizar certas complicações e melhorar o aproveitamento da oferta nutricional por meio da monitoração da TNE, na qual levará a redução da gravidade da doença, o tempo de permanência na UTI e a melhora no resultado do tratamento do paciente, bem como, minimiza os custos ${ }^{7,8}$.

Dessa forma, o objetivo do presente estudo foi avaliar o volume prescrito versus infundido em pacientes críticos com alimentação enteral exclusiva, identificando as causas de interrupções da infusão e os gastos gerados com a dieta devido a essas interrupções.

\section{METODOLOGIA}

Trata-se de um estudo observacional, 
prospectivo, realizado com pacientes internados na Unidade de Terapia Intensiva do Hospital Universitário, localizado no Município de João Pessoa (PB).

A população investigada foi todos os pacientes internados na UTI geral, de ambos os sexos, com prescrição de nutrição enteral como única fonte alimentar. Foram excluídos os pacientes menores de 18 anos, as gestantes, as puérperas e àqueles com diagnóstico clínico de morte encefálica.

A coleta de dados foi realizada no período de julho a setembro de 2019 , após a aprovação pelo Comitê de Ética em Pesquisa (CEP) do Hospital Universitário Lauro Wanderley (HULW), sob o CAAE ${ }^{\circ}$ 15144819.2.0000.5183, em 01/06/2019. Os pacientes foram acompanhados desde o início da TNE até a alta ou óbito.

Para a coleta de dados foi utilizado um formulário contendo informações dos dados pessoais do paciente (nome, gênero, idade, data de admissão e dias de internamento), diagnóstico, triagem nutricional (peso, estatura, IMC e classificação), acompanhamento dietético (tempo de jejum, tipo de administração, dieta utilizada, início e fim da terapia nutricional e volume prescrito e infundido), intercorrência (as) e motivo (os) de pausa (as) e custo de administração da dieta. A busca por essas informações foram realizados por meio das informações contidas no prontuário do paciente.

Para determinar o custo da administração da dieta por paciente, foram analisados os valores das dietas, dos equipos e dos frascos, com valores fornecidos pelo setor administrativo da nutrição.

Os dados foram organizados em pla- nilha do Excel do Programa Microsoft Office. A amostra foi delineada segundo o método de amostragem aleatória simples. A normalidade dos dados foi considerada utilizando-se o teste de normalidade de Shapiro-Wilks. Em caso de rejeição da normalidade dos dados, as comparações foram realizadas por meio do teste não paramétrico U-Mann-Whitney. Todos os testes foram realizados considerando um nível de significância estatística de $5 \%(p<0,05)$.

\section{RESULTADOS}

No presente estudo foram avaliados 27 pacientes, com idade média de 58,15 $\pm 16,21$, sendo $51,85 \%$ do gênero feminino e $48,15 \%$ do gênero masculino. Quanto ao tempo de internamento, foi observada uma média de 17,44 \pm 12,49 dias, o desvio padrão evidencia alta dispersão quando comparado ao valor médio (Tabela 1).

Com relação ao IMC, a média foi de $25,56 \mathrm{~kg} / \mathrm{m}^{2}$. Na avaliação do estado nutricional quanto à classificação do IMC, notou-se que $14,82 \%$ apresentavam-se como baixo peso, $44,44 \%$ como eutróficos e $40,74 \%$ como obesos (Tabela 1).

A Figura 1 representa a distribuição da frequência dos dias de jejum. Constatou-se que o maior percentual foi de $37,04 \%$, referente a dois dias em jejum e, $3,7 \%$ dos pacientes apresentaram quatro dias, sendo o maior valor de dias observado para a amostra.

A análise da Tabela 2 apresenta a relação entre as médias do volume total prescrito que foi de $16,53 \pm 16,4$ e o volume total infundido que foi de $13,9 \pm 13,36$. Nesse caso, notou-se uma alta variabilidade nessas variáveis, uma vez que o valor do desvio pa- 
Tabela 1. Características demográficas e antropométricas da população de estudo

\begin{tabular}{|c|c|c|c|c|c|c|c|}
\hline Variável & $\mathrm{n}$ & Média & Mediana & Mínimo & Máximo & $\begin{array}{l}\text { Desvio } \\
\text { Padrão }\end{array}$ & $\%$ \\
\hline \multicolumn{8}{|l|}{ Gênero } \\
\hline Masculino & 13 & & & & & & 48,15 \\
\hline Feminino & 14 & & & & & & 51,85 \\
\hline Idade & 27 & 58,15 & 56 & 27 & 91 & 16,21 & \\
\hline Internamento & 27 & 17,44 & 14 & 3 & 51 & 12,49 & \\
\hline IMC $\left(\mathrm{kg} / \mathrm{m}^{2}\right)$ & 27 & 25,56 & 24,1 & 16,1 & 45,1 & 7,51 & \\
\hline Classificação & & & & & & & \\
\hline Desnutrição Grau II & 02 & & & & & & 7,41 \\
\hline Desnutrição Grau I & 02 & & & & & & 7,41 \\
\hline Eutrofia & 12 & & & & & & 44,44 \\
\hline Sobrepeso & 05 & & & & & & 18,52 \\
\hline Obesidade Grau I & 01 & & & & & & 3,7 \\
\hline Obesidade Grau II & 03 & & & & & & 11,11 \\
\hline Obesidade Grau III & 02 & & & & & & 7,41 \\
\hline Total & 27 & & & & & & 100 \\
\hline
\end{tabular}

drão foi muito próximo da média, tendo sido observado que o valor do volume total estava próximo ao valor prescrito.

Na Figura 2 é apresentado o volume total prescrito e o infundido para os 27 pacientes analisados. Observou-se que existiu concordância entre os valores prescritos e os administrados. Contudo, para alguns pacientes foi possível identificar discrepâncias importantes entre essas medidas.

Foi aplicado o teste $t$ para a comparação das médias do volume prescrito versus

Figura 1. Distribuição do número de dias em jejum da população de estudo

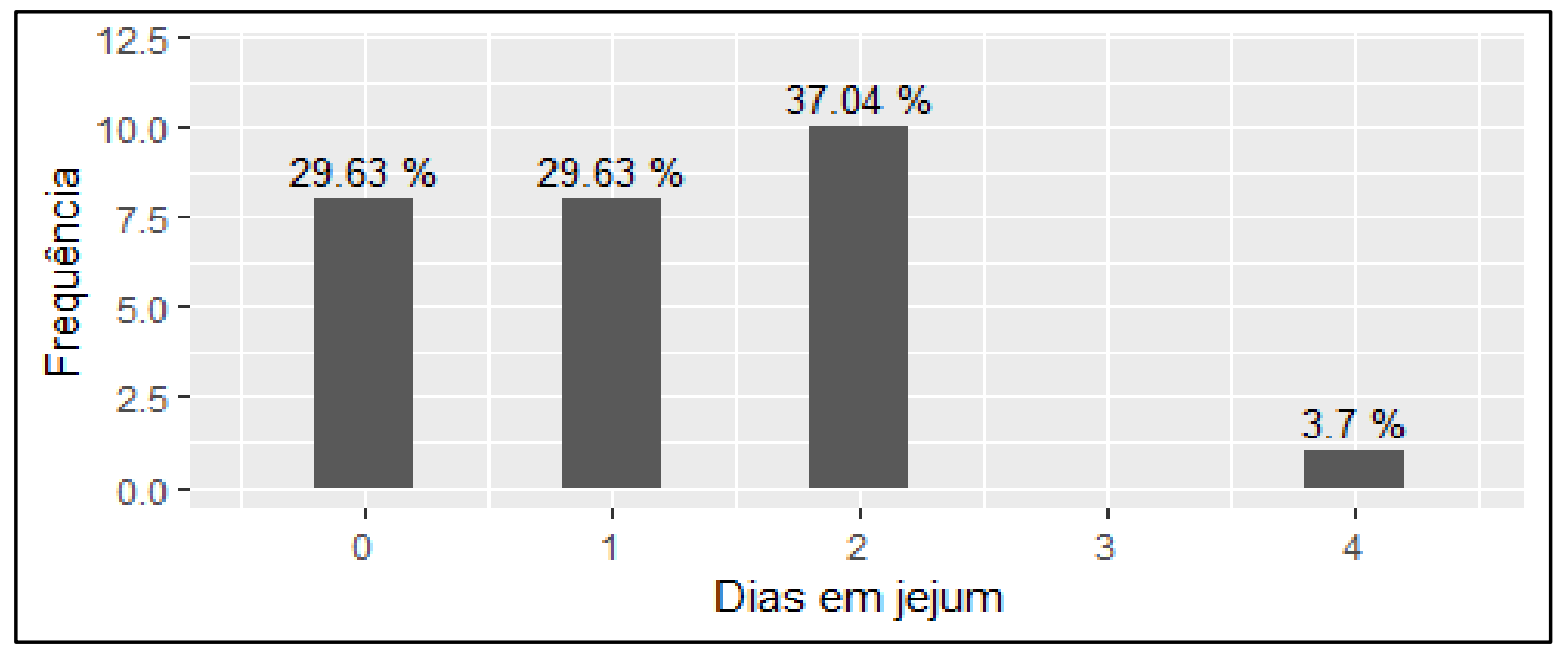


Tabela 2. Relação das médias do volume prescrito versus infundido da população de estudo

\begin{tabular}{ccccccc}
\hline Variável & & & & & & Desvio \\
& $\mathrm{n}=27$ & Média & Mediana & Mínimo & Máximo & Padrão \\
\hline Volume total prescrito(L) & 27 & 16,53 & 8,08 & 0,48 & 52,74 & 16,4 \\
Volume total infundido(L) & 27 & 13,9 & 7,28 & 0,24 & 46,32 & 13,36 \\
\hline
\end{tabular}

${ }^{*}$ Diferença estatisticamente significativa $(p<0,05)$.

Figura 2. Relação do volume prescrito versus volume infundido da população de estudo

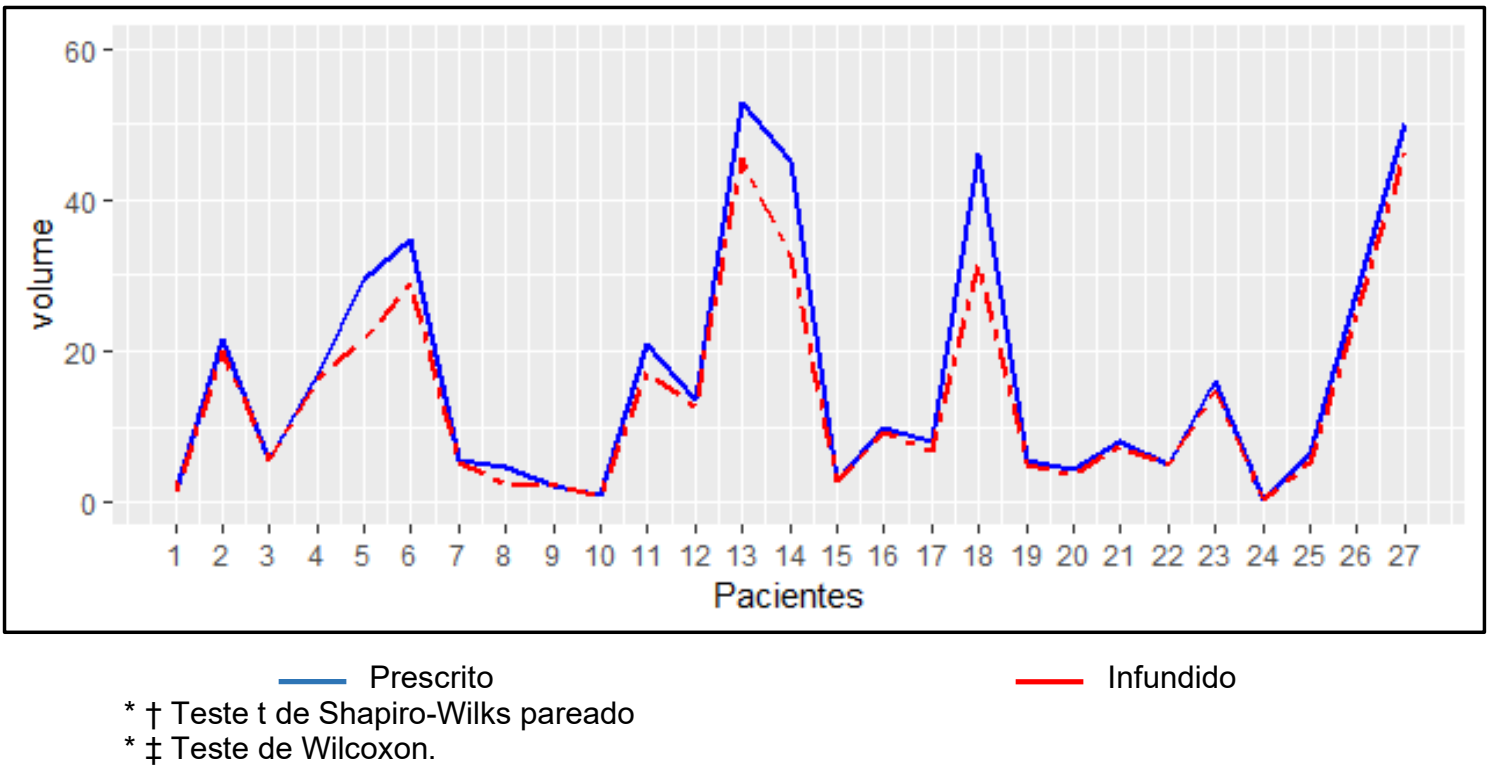

infundido. Os valores encontrados não foram estatisticamente significativos, considerando o nível de significância de $5 \%(p<0,05)$.

No tocante às intercorrências, 21 pacientes apresentaram ao menos uma intercorrência, as quais foram motivos para a interrupção da dieta. Observou-se que a diarreia, os exames e os procedimentos médicos ou da enfermagem foram os que apresentaram uma maior frequência com $25,93 \%$, cada um (Tabela 3).

Com relação à análise do valor médio total da dieta, dos equipos e dos frascos, notou-se que, para as dietas, foram de 761,49 $\pm 922,55$, para os frascos e equipos foram de $74,35 \pm 77,12$ e $2.385,78 \pm 2.135,75$, respectivamente. Considerando os gastos de forma agregada, tem-se um valor médio de 3.221,62 $\pm 3.032,64$. Observou-se, em média, um gasto superior a 2.204 reais por paciente.

\section{DISCUSSÃO}

No presente estudo a população avaliada foi majoritariamente de adultos e do gênero feminino. Um estudo realizado com pacientes críticos no hospital universitário de Petrolina (PE) observou que $71,3 \%$ dos parti- 
Tabela 3. Distribuição das intercorrências em relação às dietas enterais

\begin{tabular}{|c|c|c|}
\hline Variável & $\mathrm{n}$ & $\%$ \\
\hline \multicolumn{3}{|l|}{ Intercorrências } \\
\hline Sim & 21 & 77,78 \\
\hline Não & 06 & 22,22 \\
\hline \multicolumn{3}{|l|}{ Diarreia } \\
\hline Sim & 07 & 25,93 \\
\hline Não & 20 & 74,07 \\
\hline \multicolumn{3}{|l|}{ Vômito } \\
\hline Sim & 06 & 22,22 \\
\hline Não & 21 & 77,78 \\
\hline \multicolumn{3}{|l|}{ Exames } \\
\hline Sim & 07 & 25,93 \\
\hline Não & 20 & 74,07 \\
\hline \multicolumn{3}{|c|}{ Procedimentos médico ou da enfermagem } \\
\hline Sim & 07 & 25,93 \\
\hline Não & 20 & 74,07 \\
\hline \multicolumn{3}{|l|}{ Instabilidade hemodinâmica } \\
\hline Sim & 06 & 22,22 \\
\hline Não & 21 & 77,78 \\
\hline Total & 27 & 100 \\
\hline
\end{tabular}

Tabela 4. Descrição dos gastos da população de estudo

\begin{tabular}{lcccccc}
\hline \multicolumn{1}{c}{ Variável } & $\mathrm{n}$ & Média & Mediana & Mínimo & Máximo & Desvio \\
\hline Vadror total da dieta (R\$) & 27 & 761,49 & 450,08 & 15,48 & $4.388,21$ & 922,55 \\
Valor total do frasco (R\$) & 27 & 74,35 & 46,29 & 3,32 & 303,28 & 77,12 \\
Valor total do equipo (R\$) & 27 & $2.385,78$ & 1.408 & 176 & 8.624 & $2.135,75$ \\
\hline Total (R\$) & 27 & $3.221,62$ & 2.204 & 251,84 & $13.315,5$ & $3.032,64$ \\
\hline *Diferen
\end{tabular}

${ }^{*}$ Diferença estatisticamente significativa $(p<0,05)$.

cipantes eram do gênero masculino e $28,7 \%$ do gênero feminino, com predominância de pacientes adultos, com média de 48,5 anos ${ }^{9}$. Ao analisar os pacientes de uma UTI no hospital de câncer de Mato Grosso, foi constatado que $74 \%$ eram homens e $26 \%$ mulheres $^{10}$.

Em relação ao tempo médio de interna- ção, nesse estudo, foi menor do que o evidenciado em outros com pacientes em unidade de terapia intensiva, na qual se verificou uma média de 21,6 e 36,6 dias ${ }^{11,12}$, respectivamente.

Nos pacientes críticos existem dificuldades inerentes à avaliação do estado 
nutricional, pois os valores predeterminados para os distintos compartimentos corporais podem estar alterados. A presença de edema, as alterações nas concentrações plasmáticas de proteínas, o estado de hidratação e as mudanças no metabolismo refletem em medidas antropométricas alteradas, dificultando assim a interpretação dos resultados e, consequentemente, a avaliação do estado nutricional ${ }^{13}$.

A antropometria e o exame físico devem ser realizados de forma adequada, sistemática e progressiva para avaliar o estado nutricional do paciente crítico e, assim, estimar riscos de mortalidade e morbidade, identificando e individualizando a melhor terapia nutricional. O índice de massa corpórea (IMC) é um indicador simples do estado nutricional e, assim como os outros métodos, também têm suas limitações quando se trata de pacientes críticos. São indicativos de desnutrição, os índices inferiores a $20 \mathrm{~kg} / \mathrm{m}^{2}$, estando associados a um aumento significativo de mortalidade em diferentes tipos de pacientes. Os valores entre 18,5 e $24,9 \mathrm{~kg} /$ $\mathrm{m}^{2}$ são considerados como eutróficos. Perdas não intencionais da massa corporal maiores do que $10 \%$ ou perdas mais rápidas em 6 meses são prognósticos da evolução clínica de desnutrição, apesar da dificuldade de determinar a real perda nesses pacientes ${ }^{14}$.

No presente estudo, verificou-se que a maioria dos pacientes estava eutróficos e com excesso de peso. Em estudo conduzido em uma UTI no Rio Grande do Sul, também encontrou o mesmo resultado, sendo $16,7 \%$ em estado de magreza, 44,44\% em eutrofia e $38,9 \%$ com excesso de peso ${ }^{14}$. Ao estudar pacientes em terapia intensiva observou a presença de baixo peso em $15,36 \%$ de sobrepeso em $23,06 \%$ e de eutrofia em $61,52 \%{ }^{15}$.
A introdução precoce da nutrição enteral tem sido associada a menor tempo de permanência na UTI e a menores taxas de complicações infecciosas. As diretrizes preconizam a introdução precoce da TNE em pacientes críticos estáveis hemodinamicamente, nas primeiras 24 a 48h decorridas da admissão ${ }^{4}$. Observou-se que na população estudada o tempo desde a admissão até o início da TNE, permaneceu dentre o proposto pelas diretrizes. No estudo com pacientes críticos com terapia nutricional enteral exclusiva, observou-se que $50 \%$ dos pacientes iniciaram a TNE antes de completar 48 horas de jejum ${ }^{16}$.

Quanto ao resultado do volume prescrito versus infundido, os valores estão bem próximos, indicando uma adequação entre o que foi prescrito e o que foi administrado. No estudo sobre dieta enteral prescrita versus dieta infundida observou que esses volumes, entre os grupos etários, não ocorreram diferenças significativas. $O$ grupo de $51-80$ anos e 81-100 anos foi prescrito $804,3 \mathrm{ml}$ e 743,2 $\mathrm{ml}$, respectivamente, enquanto, o infundido foi $462,2 \mathrm{ml} \mathrm{e} \mathrm{417,0} \mathrm{ml}$, respectivamente ${ }^{17}$. Outra pesquisa verificou que os valores do volume prescrito e infundido tiveram uma diferença significativa entre os pacientes internados, em média de 1043,1 $\mathrm{ml}$ e $766,7 \mathrm{ml}$, respectivamente ${ }^{16}$, corroborando com o presente estudo.

Vários são os fatores que dificultam para um adequado aporte nutricional. Procedimentos de rotina da UTI como: jejum para exames e procedimentos, rotinas com o paciente, procedimentos como extubação, repassagem da sonda, traqueostomia, intervenções cirúrgicas, fatores relacionados à intolerância gastrointestinal e instabilidade hemodinâmica são significativos impedimentos para uma adequada nutrição enteral ${ }^{18}$. 
Outro importante fator que deve ser levado em consideração é a hiperalimentação desses pacientes, que é mais prejudicial que a subalimentação. Isso pode causar alterações metabólicas graves, diarreia, desidratação e alterações eletrolíticas, podendo resultar em graves problemas na função do sistema imune $^{19}$.

Neste estudo, observou-se que os principais motivos para a interrupção da dieta foram: diarreia, exames e procedimentos médicos ou da enfermagem. Em outros estudos, as maiores intercorrências foram: a diarreia (40\%), procedimentos da enfermagem e a bomba de infusão em (15\%) dos pacientes ${ }^{17}$. O resíduo gástrico (12\%), dieta zero para exames $(19,23 \%)$, instabilidade hemodinâmica $(17,31 \%)$, diarreia $(7,69 \%)$, dieta zero para procedimentos $(4,81 \%)$, vômitos $(2,88 \%)$, remoção acidental da sonda $(0,96 \%)$ dos pacientes $^{20}$.

Sabe-se que os episódios de diarreia devem ser analisados e registrados, pois nem sempre as causas estão relacionadas com a administração da dieta. É preciso identificar a etiologia da diarreia para iniciar o seu tratamento ${ }^{21}$. As consequências da diarreia podem levar a infecção, problemas de pele como as ulceras de pressão e a perda de eletrólitos ${ }^{22}$.

Foi descrito pela primeira vez, em 1935, que o Clostridium difficile (CD) tem sido apontado como a principal causa de diarreia no ambiente hospitalar. Nos Estados Unidos o custo que lhe é atribuído a cada episódio varia entre US\$2470,00 a US\$3669. Tem-se aumentado o número de casos de CD devido ao uso indiscriminado da antibioticoterapia, do maior número de imunossuprimidos, favorecendo assim a disseminação dos esporos ${ }^{23}$.

Outros fatores que também podem contribuir para a diarreia são os pacientes expostos aos patógenos, medicamentos e procedimentos médicos relacionados à internação prolongada ${ }^{24}$.

Com tudo que foi exposto, percebem-se as consequências que os desvios nutricionais podem acarretar. A redução da imunidade, que levará a um aumento no risco de infecções, a presença de edemas, a redução da cicatrização de feridas, na qual, aumentará o tempo de permanência hospitalar, impulsionando assim, um aumento dos custos desses pacientes ${ }^{25,26}$.

Ao analisar o custo da administração da dieta em sua pesquisa com pacientes adultos e idosos, com prescrição de nutrição enteral exclusiva, resultou em $\mathrm{R} \$ 37.690,11$ reais, gastos com os 27 pacientes em um dia. Porém apenas, $58,63 \%$ destes gastos, foram realmente utilizados pelos pacientes e 41,37\% foram descartados ${ }^{17}$.

Estudos apontam que existe uma série de variáveis que contribuem para os custos com a nutrição, sendo no Reino Unido $£ 7,00$ por dia e nos Estados Unidos (EUA), US\$ 46,00 por dia. Além disso, a maioria dos estudos é de baixa qualidade o que dificulta uma análise mais detalhada e confiável dos dados ${ }^{6}$.

\section{CONCLUSÃO}

O presente estudo mostrou que a população predominante foi de adultos e do gênero feminino. Observou-se também que na avaliação do estado nutricional, quanto à classificação, a maioria se encontrava eutróficos.

Com relação ao tempo da admissão até o início da terapia nutricional enteral, os 
pacientes receberam a administração da dieta em até 2 dias, como recomendam as diretrizes para pacientes críticos.

Os volumes prescritos de fórmulas enterais ficaram bem próximos aos valores dos volumes infundidos, porém, em alguns pacientes foi identificado diferenças significativas nesses valores. Essa aproximação de valores é importante para a evolução do paciente.

No tocante às intercorrências, que levaram a interrupção da administração da dieta, mais da metade da população estudada teve algum tipo. A diarreia, os exames e os procedimentos médicos e/ou da enfermagem foram os mais frequentes.

Quanto ao custo médio total, tem-se um valor de $\mathrm{R} \$ 3221,62$, no qual estão inclusos custos de dietas, equipos e frascos.

A avaliação do paciente crítico é dificultosa e por isso é necessário a atuação da equipe multidisciplinar, assim como, a formação continuada desses profissionais. Com isso, são importante treinamentos constantes da equipe de assistência, a criação e a utilização de protocolos, de indicadores de qualidade, para que os erros sejam minimizados e, assim, aumentar a qualidade do atendimento. Isso é necessário para proporcionar benefícios e qualidade de vida a esses pacientes e, consequentemente, ocorrerá um menor tempo de internação e de mortalidade, levando assim uma redução de custos para o hospital.

\section{REFERÊNCIAS}

1. Cederholm T, Barazzoni R, Austin P, Ballmer P, Biolo G, Bischoff SC, et al. ESPEN guidelines on definitions and terminology of clinical nutrition. Clin Nutr. 2017; 36(1):4964 .

2. Piovacari SMF, Toledo DO, Figueiredo EJA. Equipe Multiprofissional de Terapia Nutricional - EMTN em Prática. $1^{\text {a }}$.ed. Rio de Janeiro: Editora Atheneu; 2017.

3. Rosenfeld R. Falência nutricional: conceito e base para a terapia nutricional no paciente grave. In: ROSENFELD, $R$. Terapia nutricional no paciente grave. $1^{\mathrm{a}}$.ed. São Paulo: Editora Atheneu; 2014

4. Mcclave SA, Taylor BE, Martindale RG, Warren MM, Johnson DR, Braunschweig C, et al. Society of Critical Care Medicine; American Society for Parenteral and Enteral Nutrition. Guidelines for the Provision and Assessment of Nutrition Support Therapy in the Adult Critically III Patient: Society of Critical Care Medicine (SCCM) and American Society for Parenteral and Enteral Nutrition (A.S.P.E.N.). JPEN J Parenter Enteral Nutr. 2016;40(2):159-211.

5. CHINI LS, Antunes MS, Couto CS, Azevedo SO. Protocolo clínico de terapia nutricional: Procedimentos para terapia nutricional enteral e parenteral. Hospital Universitário Antônio Pedro (HUAP). Faculdade Federal Fluminense. Rio de Janeiro. 2014;2.

6. Toledo D, Castro M. Terapia nutricional na UTI. $1^{\text {a }}$.ed. Rio de Janeiro: Editora Rúbio; 2015.

7. Silveira RE, Santos AS, Sousa MC, Monteiro TSA. Gastos relacionados a hospitalizações de idosos no Brasil: perspectivas de uma década. São Paulo: Editora Einstein (São Paulo). 2013;11(4):514-520.

8. Anjos Júnior LA, Da Silva Rosa R, Reis JB, Pegoraro VA Caporossi C. Terapia nutricional enteral em pacientes críticos: qual o papel do enfermeiro nesse processo? Coorte Revista Científica do Hospital Santa Rosa. 2014; (4):53-59.

9. Santos HVD, Araujo IS. Impacto do aporte proteico e do estado nutricional no desfecho clínico de pacientes críticos. Rev. bras. ter. intensiva. 2019; 31(2):210-216.

10. Almeida FSB, DOS Santos LV, Mozer LS, DA Silva Cruz LM, DOS Santos ML, Machado PPA. Correlação entre indicadores de qualidade em terapia nutricional e estado nutricional. Mostra de Trabalhos do Curso de Nutrição do Univag. 2019; v.4.

11. Cervo AS, Magnago TSBS, Carollo JB, Chagas BP, Oliveira AS, Urbanetto JS. Eventos adversos relacionados ao uso de terapia nutricional enteral. Rev. Gaúcha Enferm. 2014;35(2):53-59

12. Rocha JB, Barbosa LS. Prescrição versus administração de dietas enterais em unidades de terapia intensiva de um hospital público de Goiás. Brasília Med. 2014;51(2):117122. 
13. Stefanello MD, Poll FA. Estado nutricional e dieta enteral prescrita e recebida por pacientes de uma Unidade de Terapia Intensiva. ABCS Health Sciences. 2014;39(2):7176.

14. Kamimura MA, Baxmann A, Sampaio L. In: Cuppari L. Guia de nutrição: nutrição clínica do adulto. $2^{\mathrm{a}}$.ed. São Paulo: Editora Manole; 2002.

15. Souza MA, Mezzomo TR. Estado nutricional e indicadores de qualidade em terapia nutricional de idosos sépticos internados em uma unidade de terapia intensiva. Rev Bras Nutr Clin. 2016; 31(1):23-28.

16. Gonçalves CV, Borges LR, Orlandi SP, Bertacco RTA. Monitoramento da terapia nutricional enteral em unidade de terapia intensiva: adequação calóricoproteica e sobrevida. Braspen J. 2017; 32(4):341-346.

17. Simões SAR, Kutz NA, Barbosa MC, Porto EF, Salgueiro MMHAO. Dieta enteral prescrita versus dieta infundida. Revista de Pesquisa: Cuidado é fundamental online. 2017; 9(3):688-695.

18. Vasconcelos MIL. Aspectos práticos na atuação do nutricionista em UTI. Programa Abbott de Especialização em Terapia Intensiva, 1999.

19. Ribeiro PC. Avaliação e suporte nutricional no paciente crítico. In: Schettino G, Cardoso LF, Mattar J. Paciente crítico, diagnóstico e tratamento: Hospital Sírio-Libanês. $2^{\mathrm{a}}$.ed. São Paulo: Editora Manole; 2006.

20. Martins TF, Campêlo WF, Vasconcelos CMCS, Henriques EMV. Avaliação da terapia nutricional enteral em pacientes críticos de uma unidade de terapia intensiva. Rev Bras em Promoção da Saúde. 2017; 30(2):255-263.
21. Campos FA, Construção e validação de protocolos de terapia de nutrição enteral, [Dissertação de Mestrado em Enfermagem na Promoção da Saúde]. Fortaleza: Universidade Federal do Ceará; 2013. $p(104 p)$.

22. Castrao DLL, Freitas MM, Zaban ALRS. Terapia nutricional enteral e parenteral: complicações em pacientes críticos: uma revisão de literatura. Comun. ciênc. Saúde. 2009:65-74.

23. Silva JM. Recentes mudanças da infecção por Clostridium difficile. São Paulo: Editora Einstein (São Paulo). 2012;10(1):105-109.

24. Borges SL, Pinheiro BV, Pace FHL, Chebli JMF. Diarréia nosocomial em unidade de terapia intensiva: incidência e fatores de risco. Arq. Gastroenterol. 2008; 45(2):117-123.

25. Fidelix MSP, DE França Santana AF, GOMES JR. Prevalência de desnutrição hospitalar em idosos. Revista da Associação Brasileira de Nutrição-RASBRAN. 2013; $5(1): 60-68$.

26. Waitzberg DL, Caiaffa WT, Correia MI. Hospital malnutrition: the Brazilian national survey (IBRANUTRI): a study of 4000 patients. Nutrition. 2001;17(7-8):573-80.

\section{CORRESPONDÊNCIA}

Mirela Ribeiro Santos Silva

Rua Rita Carneiro Diniz, 53. Bloco: B;

Apt: 203. CEP: 58077-174.

Cuiá. João Pessoa, Paraíba, Brasil.

Email: mirelaribnutri@outlook.com 